\title{
Procedimiento de Hartmann vs resección y anastomosis primaria en peritonitis diverticular de colon izquierdo por cirugía abierta. Revisión sistemática de la literatura*
}

\author{
Drs. CARMEN SANTANDER A. ${ }^{1,2}$, PAULA ASTUDILlO D. ${ }^{2,3}$, CARLOS MANTEROLA D..$^{2,3}$
}

1 Departamento de Cirugía, Universidad de Concepción.

2 Programa de Magíster en Cs. Médicas, Universidad de La Frontera.

3 Departamento de Cirugía y Traumatología, Universidad de La Frontera.

Chile.

\begin{abstract}
Hartmann's procedure and resection with primary anastomosis in diverticular peritonitis
\end{abstract}

Introduction: Colonic diverticulosis, as diverticulitis, is a frequent disease in different stages of evolution. There is uncertainty about treatment options that are used in secondary peritonitis. The aim of this study is to determine the best treatment option for patients with peritonitis secondary to diverticulitis of the left colon in terms of postoperative morbidity (POM) and mortality, comparing Hartmann's procedure (HP) and resection with primary anastomosis (RPA). Material and Methods: Systematic review. Studies in adults with peritonitis secondary to diverticulitis of the left colon treated with HP and RPA published between 1990 and 2011 were analyzed. TRIPDATABSE, IWO, MEDLINE, SciELO and LILACS databases were consulted and search strategies were applied using MeSH and free terms. Selected studies were analyzed using a score of methodological quality (MQ). The following variables were considered: mortality, POM, hospital stay, percentage of bowel transit reconstitution in patients undergoing HP and MQ of primary studies. Results: 26 primary studies were analyzed ( 47 series). There were no significant differences in the variable mortality $(p=0.0805)$, but significant difference was observed in POM (incompletely reported) $(p=0.0187)$. The median of MQ of the studies was 11 points for HP series and 10 for RPA series. Conclusion: The available evidence to determine the best treatment option in terms of mortality and POM in this kind of patients is insufficient. Studies with better level evidence and MQ are needed to clarify the uncertain.

Key words: "Diverticulitis, colonic"[Mesh], "Peritonitis"[Mesh], Hartmann 's procedure, Colonic resection, primary anastomosis, "Meta-Analysis as Topic"[Mesh].

\section{Resumen}

Introducción: La enfermedad diverticular del colon es una entidad frecuente, como también la diverticulitis en sus diferentes estadios de evolución. Existe incertidumbre respecto de las opciones terapéuticas

*Recibido el 30 de octubre de 2012 y aceptado para publicación el 22 de noviembre de 2012.

Los autores no refieren conflictos de interés.

Correspondencia: Dr. Carlos Manterola D.

Manuel Montt 112, oficina 408, Temuco, Chile. Fax: 56-45-325761.

cmantero@ufro.cl 
que se utilizan en el tratamiento de la peritonitis diverticular de colon izquierdo (PDCI). El objetivo de este estudio es determinar la mejor opción de tratamiento para pacientes con PDCI entre procedimiento de Hartmann $(\mathrm{PH})$ y resección con anastomosis primaria (RAP), en términos de mortalidad y morbilidad postoperatoria (MPO). Material y Método: Revisión sistemática de la literatura. Se analizaron estudios realizados en adultos con PDCI tratados con PH y RAP, publicados entre 1990 y 2011. Se consultó en las bases de datos TRIPDATABSE, IWO, MEDLINE, SciELO y LILACS, utilizando estrategias de búsqueda con términos $\mathrm{MeSH}$, palabras libres y operadores booleanos. Los estudios seleccionados fueron analizados mediante un escore de calidad metodológica (CM). Se consideraron las variables mortalidad, MPO, estadía hospitalaria, porcentaje de reconstitución de tránsito en pacientes sometidos a PH y CM de los estudios primarios. Resultados: Se analizaron 26 estudios primarios ( 47 series de pacientes). No se encontraron diferencias significativas respecto de la variable mortalidad $(\mathrm{p}=0,0805)$; pero sí en la variable MPO, reportada de forma incompleta $(\mathrm{p}=0,0187)$. La mediana de la CM de los estudios primarios fue de 11 puntos para las series de $\mathrm{PH}$ y de 10 para las de RAP. Conclusión: La evidencia disponible no permite determinar la mejor alternativa terapéutica en términos de mortalidad y MPO en este tipo de pacientes. Se requieren estudios de mejor nivel de evidencia y CM para aclarar esta incertidumbre.

Palabras clave: Diverticulitis colónica, peritonitis, operación de Hartmann, resección de colon, anastomosis primaria, revisión sistemática de la literatura.

\section{Introducción}

La enfermedad diverticular del colon (EDC), es una patología frecuente en los países desarrollados y es causa de un número importante de hospitalizaciones y utilización de recursos médicos ${ }^{1-3}$. La incidencia estimada de diverticulitis colónica es de 10 pacientes/100.000/año ${ }^{4}$. En 2004 en EE.UU., la EDC fue causa de 312.000 hospitalizaciones y su frecuencia aumentó en $26 \%$ en 10 años ${ }^{1}$.

Existe evidencia que un $20 \%$ a $30 \%$ de los pacientes con EDC experimentarán al menos un episodio de diverticulitis aguda ${ }^{4-7}$. La mayoría de estos casos evolucionan favorablemente con tratamiento médico ${ }^{8}$; sin embargo, hasta el $25 \%$ de estos pacientes requerirán intervenciones quirúrgicas de urgencia debido a perforación diverticular con peritonitis difusa; situación que se asocia a riesgo de mortalidad y morbilidad ${ }^{4,6,7-9}$.

El tratamiento estándar de la peritonitis aguda por EDC izquierdo es la resección del segmento colónico comprometido y la colostomía terminal del segmento proximal, conocido como procedimiento de Hartmann $(\mathrm{PH})^{6,7,10}$. Este, se asocia a morbilidad de hasta $60 \%$ y mortalidad de hasta $30 \%{ }^{11,12-14}$; y requiere de una segunda operación para reconstituir el tránsito intestinal, de la que se reportan cifras de mortalidad y morbilidad postoperatoria (MPO) de hasta $4 \%$ y $30 \%$ respectivamente ${ }^{11,12-15}$. Por otro lado, hay evidencia que alrededor de $30 \%$ a $50 \%$ de pacientes sometidos a un PH no se les reconstituye el tránsito ${ }^{11-17}$.

Existe evidencia tipo IV, en resección y anastomosis primaria (RAP) por peritonitis diverticular de colon izquierdo (PDCI), sin utilización de ostomía de protección ${ }^{10,12-14,18-31}$. Sin embargo, en los consensos americano y europeo no se estableció una indicación clara al respecto.
El objetivo de este estudio es determinar la mejor opción terapéutica para la PDCI en términos de mortalidad y MPO, al comparar la RAP y PH.

\section{Material y Método}

\section{Diseño}

Revisión sistemática de la literatura.

\section{Población}

Estudios primarios cuya población correspondiera a sujetos mayores de 18 años con diagnóstico de diverticulitis aguda de colon izquierdo asociado a peritonitis difusa o fecaloídea, sometidos a $\mathrm{PH}$ o RAP; cuyos diseños fueran ensayos clínicos, estudios de cohorte, de casos y controles, corte transversal o series de casos; publicados entre enero de 1990 y junio de 2011; sin restricción idiomática. Se excluyeron estudios contaminados con pacientes con neoplasias y trauma de colon; y estudios cuya población fuesen pacientes sometidos a cirugía colónica videolaparoscópica.

\section{Definición}

Se definió PDCI a aquella peritonitis generalizada purulenta y fecaloídea, grados 3 y 4 de la clasificación de Hinchey ${ }^{32}$.

\section{Metodología de búsqueda}

Se realizó con base a los componentes básicos PICoR: población de interés $(\mathrm{P})$, intervención que se desea valorar (I), el comparador para la intervención en estudio (Co), y la variable resultado que se medirá a partir de la intervención en estudio (R). A partir de esta estrategia se buscaron estudios primarios que versaran sobre pacientes con diverticulitis aguda y 
PDCI (P), intervenidos con PH (I), que se comparara con RAP (Co) y cuya variable de respuesta fuese mortalidad y MPO (R). Para ello, se revisaron las siguientes bases de datos: TRIP database, ISI Web of Knowledge, MEDLINE, SciELO y LILACS; utilizando términos MeSH, palabras libres y los conectores booleanos AND y OR; estrategias que se adaptaron para cada una de las bases de datos. Por ejemplo, para MEDLINE, se generó el comando [(Colonic diverticulitis) OR (Peritonitis) OR (Diverticulitis, colonic(Mesh)) OR (Peritonitis(Mesh))] AND [(Hartmann's procedure) OR (Colonic resection) OR (Ostomy) OR(Surgical procedures, operative(Mesh)) OR (Surgical stomas(Mesh)] AND [(Primary anastomosis) OR (Anastomosis, surgical(Mesh)) OR (Surgical procedures, operative(Mesh)] AND [(mortality) OR (mortality(Mesh)) OR (surgical wound infection) OR (anastomotic leak) OR (Postoperative complications(Mesh)) OR (Surgical wound infection(Mesh)) OR (Anastomotic leak(Mesh)) OR(Surgical wound dehiscence(Mesh))].

\section{Variables de medición}

Las variables de resultado primarias fueron mortalidad y MPO. Las variables de resultado secundarias fueron estancia hospitalaria, frecuencia de reconstitución del tránsito y morbi-mortalidad asociada; y CM los estudios primarios. Esta última, se determinó aplicando la propuesta MINCIR terapia (escala que valora los estudios con 6 a 36 puntos, siendo el punto de corte de 18 puntos (o más), el utilizado para considerar un estudio de CM adecuada $)^{33-35}$. Este instrumento se aplicó de forma enmascarada por dos investigadores independientes (CS y PA), los que resolvieron sus diferencias por consenso.

\section{Plan de análisis}

Recolección de datos de los estudios primarios seleccionados por dos revisores mediante la confección de base de datos en Windows Excel según técnica quirúrgica y variables resultado. Se aplicó estadística descriptiva, con cálculo de medidas de tendencia central y dispersión. Posteriormente, se aplicó a los artículos primarios seleccionados el escore MINCIR de CM. Luego, se calcularon los promedios ponderados, fórmula que permite ponderar los resultados de los artículos primarios en relación a la CM de cada uno de ellos, de manera de que los resultados sean los más cercanos a la realidad ${ }^{34,35}$ (Figura 1). Se valoró la heterogeneidad de los artículos primarios aplicando el test Q de Cochrane; finalmente, se aplicó T-test para comparar promedios ponderados y pruebas no paramétricas (KruskalWallis), para comparar medianas.

\section{Aspectos éticos}

Durante el análisis de los artículos primarios seleccionados se realizó enmascaramiento de autores, centros de estudio y títulos. De esta manera se intentó evitar los sesgos de selección y análisis. Se declara la no existencia de conflictos de interés por parte de los autores.

\section{Resultados}

Al aplicar los términos de búsqueda definidos en cada base se obtuvo un total de 2.596 artículos, de los cuales se seleccionan por título atingente a la pregunta de investigación un total de 234 (Tabla 1). Se descartaron 208 artículos por diversos criterios; por lo que quedaron 26 artículos para el análisis final $1^{11,18,19,24-26,36-38,40,43,44,46-59}$, los que se transformaron según el tipo de diseño, en 47 series de pacientes (24 series de PH y 23 de RAP) (Figura 2).

Los artículos seleccionados fueron publicados entre 1990 y 2011, sin mayor frecuencia para un año determinado (mediana en el año 2002; aunque el $76,9 \%$ de ellos fueron publicados entre los años 2000 y 2011).

Tres artículos $(11,5 \%)$, corresponden a series de casos de PH, 2 estudios $(7,7 \%)$ a series de casos de

Tabla 1. Referencias bibliográficas obtenidas según bases de datos consultadas

\begin{tabular}{|lcc|}
\hline Fuente & $\begin{array}{c}\text { Artículos } \\
\text { obtenidos }\end{array}$ & $\begin{array}{c}\text { Artículos } \\
\text { seleccionados }\end{array}$ \\
\hline TRIP database & 312 & 9 \\
ISI Web of knowledge & 805 & 72 \\
MEDLINE & 1.273 & 118 \\
SciELO & 56 & 7 \\
LILACS & 150 & 28 \\
Total & 2.596 & 234 \\
\hline
\end{tabular}

\section{$\mathrm{PP}_{\mathrm{GT}}=\underline{\Sigma \mathrm{Xi}^{*} \mathrm{ei}}$ $\sum \mathrm{ei}$}

Figura 1. Fórmula para cálculo de promedios ponderados, en la que $\boldsymbol{X} \boldsymbol{i}$ corresponde al valor de la variable en el estudio "i" (para todas las variables en estudio); $\boldsymbol{e i}$ corresponde al escore obtenido por el estudio "i"; y $\sum \boldsymbol{e i}$ corresponde a la sumatoria de los escores de todos los estudios. 


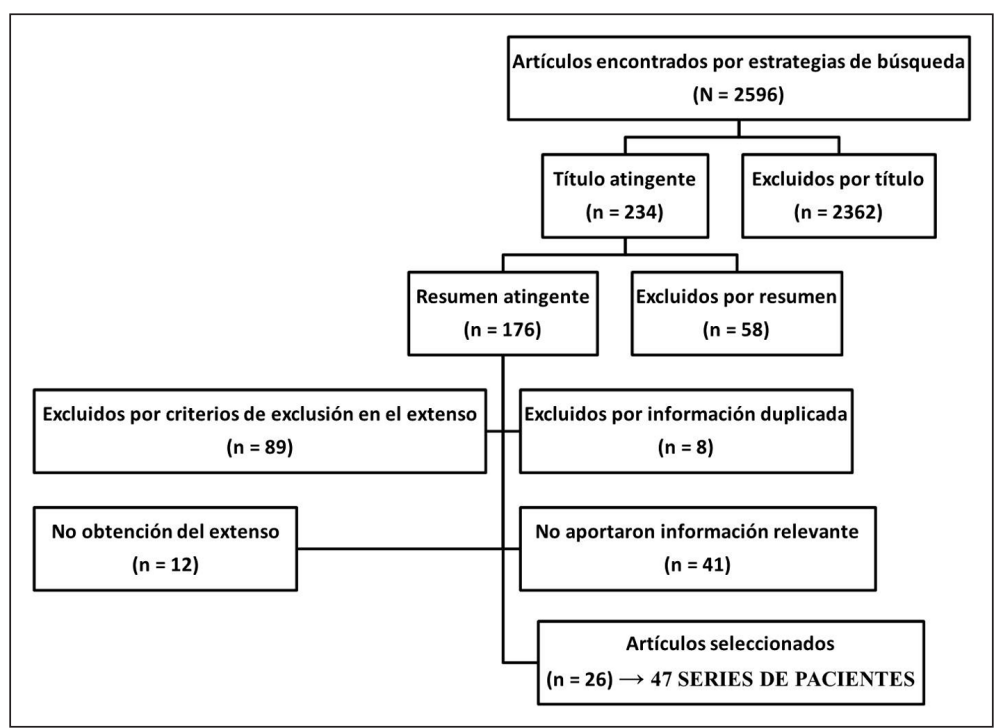

Figura 2. Flujograma de selección de estudios primarios.
RAP y 21 artículos $(80,8 \%)$ a estudios comparativos entre ambas técnicas. Todos estos estudios, representan un total de 1.342 sujetos tratados, $1.054(78,5 \%)$ de ellos con PH y $289(21,5 \%)$ con RAP. Las características de los estudios primarios se pueden observar en la Tabla 2.

Sólo 5 de las 24 series de PH y 6 de las 23 series de RAP reportaron la distribución de las características biodemográficas de las poblaciones estudiadas. Es así como la edad promedio de los pacientes tratados con PH fue de 68,7 años y la de aquellos tratados con RAP de 65,3 años. La totalidad de los estudios primarios reportaron mortalidad, variable que al ponderarla según CM de los artículos que la originaron generaron una diferencia de $7 \%$ menor para RAP. La MPO fue reportada en 6 de las 24 series de $\mathrm{PH}$ y en 4 de las 23 series de RAP (los demás estudios sólo reportaron la frecuencia de causas aisladas de MPO, tales como infección de sitio operatorio y dehiscencia de herida operatoria); de este modo, se verificó una diferencia de MPO ponderada por $\mathrm{CM}$ de $27 \%$, menor para RAP. El promedio ponderado de la estadía hospitalaria fue reportado por 6 de las 24 series de PH y por 7 de las 23 series de RAP, con una diferencia entre ambas de 7 días, menor para RAP (Tabla 3).

Al aplicar el escore de CM, se comprobó que los promedios de puntuación de la totalidad de los estudios primarios
Tabla 2. Características de los estudios primarios

\begin{tabular}{|c|c|c|c|c|}
\hline $\begin{array}{l}\text { Autor } \\
\text { principal }\end{array}$ & $\begin{array}{c}\text { Año de } \\
\text { publicación }\end{array}$ & $\begin{array}{l}\text { Base de da- } \\
\text { tos de origen }\end{array}$ & $\begin{array}{c}\text { Período } \\
\text { de estudio }\end{array}$ & $\begin{array}{c}\text { Escore de } \\
\text { CM }\end{array}$ \\
\hline Karoui $^{40}$ & 2009 & PM / TRIP & 1994-2006 & 10 \\
\hline Casal-Núñez ${ }^{38}$ & 2008 & PM & 1993-2007 & 12 \\
\hline Stumpf ${ }^{25}$ & 2007 & PM & $1998-2003$ & 12 \\
\hline Vermeulen $^{54}$ & 2009 & PM & $1995-2005$ & 13 \\
\hline Richter $^{43}$ & 2006 & PM & $2001-2003$ & 9 \\
\hline Regenet $^{37}$ & 2003 & ISI / PM & 1994-2001 & 13 \\
\hline Blair $^{48}$ & 2002 & PM & $1989-2000$ & 12 \\
\hline Landen $^{57}$ & 2002 & ISI / PM & 1994-1999 & 9 \\
\hline Schilling ${ }^{26}$ & 2001 & PM & 1994-1998 & 10 \\
\hline Gooszen $^{47}$ & 2001 & ISI / PM & $1979-1993$ & 11 \\
\hline $\mathrm{Lee}^{24}$ & 1997 & ISI / PM & 1987-1996 & 9 \\
\hline Tucci $^{\mathbf{5 3}}$ & 1996 & PM & 1986-1994 & 11 \\
\hline Belmonte $^{11}$ & 1996 & $\mathrm{PM}$ & 1988-1993 & 10 \\
\hline Peoples $^{36}$ & 1990 & PM & 1982-1986 & 9 \\
\hline Mäkelä55 & 2002 & PM & $1986-2000$ & 8 \\
\hline Trenti ${ }^{50}$ & 2011 & ISI / PM & $1995-2008$ & 12 \\
\hline Frileux $^{56}$ & 2010 & ISI / PM & $1995-2002$ & 9 \\
\hline Mueller ${ }^{18}$ & 2011 & ISI / PM & 1996-2006 & 11 \\
\hline Holmer $^{58}$ & 2011 & ISI & 1998-2009 & 13 \\
\hline Alvarez ${ }^{46}$ & 2007 & ISI & $1986-2005$ & 12 \\
\hline Fantozzi $^{19}$ & 2008 & LILACS & $1997-2006$ & 9 \\
\hline Hequera $^{59}$ & 2005 & LILACS & 1994-2004 & 9 \\
\hline Chandra $^{44}$ & 2004 & PM & 1983-1999 & 13 \\
\hline Thaler $^{51}$ & 2000 & PM & 1988-1998 & 12 \\
\hline Tudor ${ }^{49}$ & 1994 & PM & 1985-1988 & 8 \\
\hline Sarin $^{52}$ & 1991 & PM & $1980-1987$ & 10 \\
\hline
\end{tabular}

PM: MEDLINE. ISI: ISI Web of knowledge. TRIP: TRIP Database. 
Tabla 3. Distribución de las series según variables en estudio

\begin{tabular}{|c|c|c|c|}
\hline Tratamiento realizado & PH & RAP & $\mathbf{p}$ \\
\hline $\begin{array}{c}\text { Género }(\%) \\
\text { Femenino } \\
\text { Masculino }\end{array}$ & $\begin{array}{l}51,0 \\
49,0\end{array}$ & $\begin{array}{l}48,0 \\
52,0\end{array}$ & 0,025 \\
\hline $\begin{array}{l}\text { Edad } \\
(\text { promedio } \pm \text { DE) } \\
(\text { valores extremos) }\end{array}$ & $\begin{array}{l}68,7 \pm 2,5 \\
(66,7-72)\end{array}$ & $\begin{array}{l}65,3 \pm 5,7 \\
(58,1-72)\end{array}$ & 0,186 \\
\hline $\begin{array}{l}\text { Mortalidad }(\%) \\
\text { (promedio ponderado) }\end{array}$ & 18,0 & 11,0 & 0,0805 \\
\hline $\begin{array}{l}\text { MPO }(\%) \\
\text { (promedio ponderado) }\end{array}$ & 36,0 & 9,0 & 0,0187 \\
\hline $\begin{array}{l}\text { Estancia hospitalaria (días) } \\
\text { (promedio ponderado) }\end{array}$ & 24,0 & 17,0 & 0,199 \\
\hline $\begin{array}{l}\text { Escore MINCIR } \\
\text { (promedio } \pm \mathrm{DE}) \\
\text { (valores extremos) }\end{array}$ & $\begin{array}{c}10,7 \pm 1,7 \\
(8-13)\end{array}$ & $\begin{array}{c}10,5 \pm 1,6 \\
(8-12)\end{array}$ & 0,704 \\
\hline
\end{tabular}

PH: Procedimiento de Hartmann. RAP: Resección y anastomosis primaria. DE: Desviación estándar. MPO: Morbilidad postoperatoria.

fue de 10,7 $\pm 1,65$ puntos ( 8 a 13 puntos). Ningún artículo alcanzó el valor de corte de 18 puntos. Al agruparlos por tipo de tratamiento, los promedios de las puntuaciones fueron muy similares (Tabla 3 ).

La reconstitución de tránsito intestinal en el grupo de pacientes tratados con $\mathrm{PH}$ ocurrió en el $68,1 \%$ de las veces (obtenido del reporte de 6 de las 24 series). Por su parte, la mortalidad reportada fue de $0 \%$ (obtenido de la información de 4 de las 24 series) y la MPO de $24 \%$ (obtenido del reporte de 1 de las 24 series).

\section{Discusión}

Como se señaló anteriormente, la EDC; en especial del colon sigmoides, es una patología prevalente en países occidentales y en poblaciones con tendencia al envejecimiento; y un porcentaje no despreciable presentará una complicación inflamatoria aguda, cuyo escenario más dramático corresponde al de la peritonitis difusa, tanto purulenta como fecaloídea ${ }^{60}$. Este cuadro se da en general en pacientes mayores, con patologías asociadas y se asocia a una importante morbimortalidad perioperatoria. $\mathrm{La}$ indicación de cirugía en estos casos es clara, pero existe debate y por ende incertidumbre respecto a qué técnica elegir. El paradigma actual establece al PH como estándar de referencia, independiente de no tener un buen nivel de evidencia que lo avale. Adicionalmente, esta técnica implica la necesidad de realizar una segunda intervención para reconstituir el tránsito intestinal; la que se asocia a mortalidad y MPO per se, alterando la calidad de vida de estos pacientes, que en ocasiones deben quedar con una colostomía permanente ${ }^{61,62}$.
Con el fin de evitar estos inconvenientes se han propuesto otras alternativas, entre las que destaca la RAP, que permite resolver en un tiempo el problema. Debido a que aún existe desconfianza respecto a que la RAP ofrezca mejores resultados al paciente con PDCI en términos de mortalidad y MPO asociadas que el PH; la indicación de RAP no ha sido claramente establecida y aún queda a "criterio del cirujano" si es o no realizada.

La mayor parte de los cuadros de PDCI se dan en el contexto de urgencia, donde el cirujano general se ve en el dilema de decidir por un procedimiento cuya indicación no está establecida; razón por la que la potencial responsabilidad frente a una complicación queda en manos del cirujano tratante. Esta situación amerita aclarar la incertidumbre e intentar determinar la mejor alternativa terapéutica.

Esta revisión sistemática nos permite verificar que la evidencia disponible en relación a las técnicas en evaluación para el tratamiento de la PDCI es escasa y de baja CM (estudios primarios de nivel de evidencia tipo 4 y escore de CM inferior a 18 puntos); y además, con reporte incompleto de cada una de las variables de resultado en estudio. Por otro lado, como la mayoría de los estudios primarios no entregan información del perfil biodemográfico ni de otros factores como patologías asociadas o índices de gravedad de los pacientes, no pudimos saber si los grupos estudiados eran comparables; lo que constituye un sesgo a considerar en el análisis de los resultados.

Otra fuente posible de sesgo podría constituir el amplio período de tiempo definido para la búsqueda de estudios primarios, la cual fue definida en base a un criterio técnico, en el que consideramos que a partir de esta fecha existen avances tanto en el cuidado perioperatorio de los pacientes (desarrollo de protocolos de tratamiento de pacientes sépticos en unidades de cuidados intensivos), así como en el intraoperatorio (desarrollo de suturas mecánicas, elementos de disección ultrasónica, etc.), los que podrían influir en la mortalidad y MPO.

Intentamos disminuir sesgos al restringir los criterios de selección, excluyendo intervenciones vídeo laparoscópicas y estudios contaminados con pacientes en los que el origen de la peritonitis correspondiera a otras patologías (neoplasias y trauma). Si bien, aplicando una menor restricción a los criterios de selección podríamos haber obtenido un número significativamente mayor de artículos primarios, habríamos introducido una nueva 
fuente de sesgos al ya importante número de sesgos presentes.

El hecho de no encontrar diferencias estadísticamente significativas al comparar la mortalidad entre ambos tratamientos, puede ser interesante; sobretodo si se considera que esta variable fue reportada por la totalidad de los estudios primarios incluidos.

Por otra parte, que exista diferencia significativa en los promedios ponderados de la variable MPO, nos parece que debe interpretarse con cautela en vista que esta variable solamente fue reportada por el $21,3 \%$ de las series; lo que determina una importante fuente de sesgo de reporte.

En cuanto a las variables de resultado secundarias, la única potencialmente susceptible de comparación es la estadía hospitalaria, en la que no se observaron diferencias significativas.

El porcentaje de reconstitución de tránsito intestinal, su mortalidad y MPO asociada en sujetos tratados con $\mathrm{PH}^{61-63}$, son hechos a considerar en la toma de decisiones por alguna alternativa terapéutica en caso de obtener diferencias significativas en los promedios ponderados de las variables de resultado primarias. Sin embargo, su reporte también es incompleto (sólo el 25\% de los artículos lo reporta).

Por ello es que a modo de conclusión, se puede señalar que la evidencia actual no permite demostrar diferencias contundentes en términos de validez interna y externa entre PH y RAP para el tratamiento de la PDCI, respecto de las variables mortalidad y MPO. La insuficiente CM de los artículos disponibles hace imposible la obtención de conclusiones válidas que permitan contestar cuál es la mejor opción terapéutica y por ende formular recomendaciones; razón por la que se necesitan estudios de carácter prospectivo y de mejor CM.

\section{Referencias}

1. Etzioni DA, Mack TM, Beart RW Jr, Kaiser AM. Diverticulitis in the United States: 1998-2005: changing patterns of disease and treatment. Ann Surg. 2009;249:2107.

2. Jun S, Stollman N. Epidemiology of diverticular disease. Best Pract Res Clin Gastroenterol. 2002;16:529-42.

3. Martel J, Raskin JB, ND SG. History, incidence, and epidemiology of diverticulosis. J Clin Gastroenterol. 2008;42:1125-7.

4. Aldoori WH, Giovannucci EL, Rimm EB, Wing AL, Trichopoulos DV, Willett WC. A prospective study of alcohol, smoking, caffeine, and the risk of symptomatic diverticular disease in men. Ann Epidemiol. 1995;5:221-8.

5. Köhler L, Sauerland S, Neugebauer E. The Scientific Committee of the European Association for Endoscopic
Surgery. Diagnosis and treatment of diverticular disease: results of a consensus development conference. Surg Endosc. 1999;13:430-6.

6. Rafferty J, Shellito P, Hyman N, Buie D and the Standards Committee of The American Society of Colon and Rectal Surgeons. Dis Colon Rectum 2006;49:939-44.

7. Fozard JB, Armitage NC, Schofield JB, Jones OM. ACPGBI Position Statement on Elective Resection for Diverticulitis. Colorectal Dis. 2011;13(Suppl3):1-11.

8. Novitsky Y, Sechrist C, Payton B, Kercher K, Heniford B. Do the risks of emergent colectomy justify nonoperative management strategies for recurrent diverticulitis? Am J Surgery 2009;197:227-31.

9. Parra-Blanco A. Colonic Diverticular Disease: Pathophysiology and Clinical Picture. Digestion 2006;73(Suppl1):47-57.

10. Wong WD, Wexner SD, Lowry A, Vernava A III, Burnstein M, Denstman F, et al. Practice parameters for the treatment of sigmoid diverticulitis-supporting documentation. The Standards Task Force. The American Society of Colon and Rectal Surgeons. Dis Colon Rectum 2000;43:290-7.

11. Belmonte C, Klas JV, Pérez JJ. The Hartmann procedure. First choice or last resort in diverticular disease? Arch Surg. 1996;131:612-5.

12. Salem L, Flum DR. Primary anastomosis or Hartmann's procedure for patients with diverticular peritonitis? A systematic review. Dis Colon Rectum 2004;47:1953-64.

13. Constantinides VA, Tekkis PP, Athanasiou T. Primary resection with anastomosis vs. Hartmann's procedure in nonelective surgery for acute colonic diverticulitis: a systematic review. Dis Colon Rectum 2006;49:966-81.

14. Abbas S. Resection and primary anastomosis in acute complicated diverticulitis, a systematic review of the literature. Int J Colorectal Dis. 2007;22:351-7.

15. Wigmore SJ, Duthie GS, Young IE. Restoration of intestinal continuity following Hartmann's procedure: the Lothian experience 1987-1992. Br J Surg. 1995;82:2730 .

16. Salem TA, Molloy RG, O’Dwyer PJ. Prospective study on the management of patients with complicated diverticular disease. Colorectal Dis. 2006;8:173-6.

17. Dias A, Gondim A, Nahas S. Atualização no tratamento da diverticulite aguda do cólon/Recent evidences in the management of acute diverticulitis Rev Bras Coloproctol. 2006;29:363-71.

18. Mueller M, Karpitschka M, Renz B, Kleespies A, Kasparek M, Jauch K, ET al. Co-morbidity and postsurgical outcome in patients with perforated sigmoid diverticulitis. Int J Colorectal Dis. 2011;26:227-34.

19. Fantozzi M, Vecchio P, Lococo, J, Dezanzo V. Tratamiento quirúrgico de la peritonitis purulenta generalizada de origen diverticular. Rev Argent Coloproctol 2008;19:79-88.

20. Alvarez G, Mazzurana M. Diverticulite aguda complicada tratada por cirurgia laparoscópica assistida com a 
mão (Hals): descrição da técnica e revisão da literatura. Rev Bras Colo-proctol. 2006;26:275-9.

21. Constantinides VA, Tekkis PP, Senapati A. Association of Coloproctology of Great Britain Ireland. Prospective multicentre evaluation of adverse outcomes following treatment for complicated diverticular disease. $\mathrm{Br} \mathrm{J}$ Surg. 2006;93:1503-13.

22. Constantinides V, Heriot A, Remzi F, Darzi A, Senapati A, Fazio V, et al. Operative Strategies for Diverticular Peritonitis. A Decision Analysis Between Primary Resection and Anastomosis Versus Hartmann's Procedures. Ann Surg. 2007;245:94-103.

23. Issa N, Dreznik Z, Dueck D, Arish A, Ram E, Kraus M, et al. Emergency Surgery for complicated acute diverticulitis. Colorectal Disease 2009;11:198-202.

24. Lee E, Murray J, Coller J, Roberts P, Schoetz D. Intraoperative Colonic Lavage in Nonelective Surgery for Diverticular Disease. Dis Colon Rectum 1997;40:66974.

25. Stumpf M, Vinces F, Edwards J. Is Primary Anastomosis Safe in the Surgical Management of Complications of Acute Diverticulitis? Am Surgeon 2007;73:787-91.

26. Schilling M, Maurer C, Kollmar O, Buchler M. Primary vs. secondary anastomosis after sigmoid colon resection for perforated diverticulitis (Hinchey Stage III and IV): a prospective outcome and cost analysis. Dis Colon Rectum 2001;44:699-703.

27. Vermeulen J, Akkersdijk GP, Gosselink MP, Hop WC, Mannaerts GH, van der Harst E, et al. Outcome after emergency surgery for acute perforated diverticulitis in 200 cases. Dig Surg. 2007;24:361-6.

28. Gooszen A, Tollenaar R, Geelkerken R, Smeets H, Bemelman W, Van Schaardenburgh P, et al. Prospective study of primary anastomosis following sigmoid resection for suspected acute complicated diverticular disease. Br J Surg. 2001;88:693-7.

29. Hoemke M, Treckmann J, Schmitz R, Shah S. Complicated diverticulitis of the sigmoid: a prospective study concerning primary resection with secure primary anastomosis. Dig Surg. 1999;16:420-4.

30. Krukowski ZH, Matheson NA. Emergency surgery for diverticular disease complicated by generalized and faecal peritonitis: a review. Br J Surg. 1984;71:921-7.

31. Zeitoun G, Laurent A, Rouffet F, Hay JM, Fingerhut A, Paquet J, et al. Multicentre, randomized clinical trial of primary versus secondary sigmoid resection in generalised peritonitis complicating sigmoid diverticulitis. $\mathrm{Br}$ J Surg. 2000;87:1366-74.

32. Hinchey EJ, Schaal PG, Richards GK. Treatment of perforated diverticular disease of the colon. Adv Surg. 1978;12:85-109.

33. Manterola C, Pineda V, Vial M, Losada H, Muñoz S. Revisión sistemática de la literatura. Propuesta metodológica para su realización. Rev Chil Cir. 2003;55:204-8.

34. Manterola C, Vial M, Pineda V, Sanhueza A. Revisión Sistemática de la Literatura con Diferentes Tipos de
Diseños. Int J Morphol. 2009;27:1179-86.

35. Manterola C, Cortés M. Cómo valorar e interpretar un artículo sobre tratamiento o procedimientos terapéuticos. Rev Chil Cir. 2010;62:639-48.

36. Peoples J, Vilk D, Maguire J, Elliott D. Reassessment of primary resection of the perforated segment for severe colonic diverticulitis. Am J Surg. 1990;159:291-3.

37. Regenet N, Pessaux P, Hennekinne S, Lermite E, Tuech $\mathrm{J}$, Brehant $\mathrm{O}$, et al. Primary anastomosis after intraoperative colonic lavage vs. Hartmann's procedure in generalized peritonitis complicating diverticular disease of the colon. Int J Colorectal Dis. 2003;18:503-7.

38. Casal Núñez JE, Ruano Poblador A, García Martínez MT, Carracedo Iglesias R, Del Campo Pérez V. Morbidity and mortality after a Hartmann operation due to peritonitis originating from a sigmoid diverticulum disease (Hinchey grade III-IV). Cir Esp. 2008;84:210-4.

39. Regenet N, Tuech J, Pessaux P, Ziani M, Rouge C, Hennekinne $\mathrm{S}$, et al. Intraoperative colonic lavage with primary anastomosis vs. Hartmann's procedure for perforated diverticular disease of the colon: a consecutive study. Hepatogastroenterology 2002;49: 664-7.

40. Karoui M, Champault A, Pautrat K, Valleur P, Cherqui D, Champault G. Laparoscopic peritoneal lavage or primary anastomosis with defunctioning stoma for Hinchey 3 complicated diverticulitis: results of a comparative study. Dis Colon Rectum 2009;52:609-15.

41. Chouillard E, Maggiori L, Ata T, Jarbaoui S, Rivkine E, Benhaim L, et al. Laparoscopic two-stage left colonic resection for patients with peritonitis caused by acute diverticulitis. Dis Colon Rectum 2007;50:1157-63.

42. Zingg U, Pasternak I, Dietrich M, Seifert B, Oertli D, Metzger U. Primary anastomosis vs Hartmann's procedure in patients undergoing left colectomy for perforated diverticulitis. Colorectal Dis. 2010;2:54-60.

43. Richter S, Lindemann W, Kollmar O, Pistorius G, Maurer $\mathrm{C}$, Schilling $\mathrm{M}$.One stage sigmoid colon resection for perforated diverticulitis (Hinchey stages III and IV). World J Surg. 2006;30:1027-32.

44. Chandra V, Nelson H, Larson D, Harrington J. Impact of primary resection on the outcome of patients with perforated diverticulitis. Arch Surg. 2004;139:1221-4.

45. Biondo S, Perea MT, Ragué JM, Parés D, Jaurrieta E. One-stage procedure in non-elective surgery for diverticular disease complications. Colorectal Dis. 2001;3:425.

46. Alvarez JA, Baldonedo RF, Bear IG, Otero J, Pire G, Alvarez $\mathrm{P}$, et al. Presentation, management and outcome of acute sigmoid diverticulitis requiring hospitalization. Dig Surg. 2007;24:471-6.

47. Gooszen AW, Gooszen HG, Veerman W, Van Dongen VM, Hermans J, Klein E, et al. Operative Treatment of Acute Complications of Diverticular Disease: Primary or Secondary Anastomosis after Sigmoid Resection. Eur J Surg. 2001;167:35-9.

48. Blair N, Germann M. Surgical management of acute 
sigmoid diverticulitis. Am J Surg. 2002;183:525-8.

49. Tudor R, Farmakis N, Keighley M. National audit of complicated diverticular disease: analysis of index cases. Br J Surg. 1994;81:730-2.

50. Trenti L, Biondo S, Golda T, Millan M, Kreisler E, Fraccalveri D, et al. Generalized peritonitis due to perforated diverticulitis: Hartmann's procedure or primary anastomosis? Int J Colorectal Dis. 2011;26:377-84.

51. Thaler K, Neumann F, Gerö A, Kreuzer W. Utility of appropriate peritonitis grading in the surgical management of perforated sigmoid diverticulitis. Colorectal Dis. 2000;2:359-63.

52. Sarin S, Boulos P. Evaluation of current surgical management of acute inflammatory diverticular disease. Ann Royal Coll Surg Engl. 1991;73:278-82.

53. Tucci G, Torquati A, Grande M, Stroppa I, Sianesi M, Farinon A. Major Acute Inflammatory Complications of Diverticular Disease of the Colon: Planning of Surgical Management. Hepatogastroenterol. 1996;43:839-45.

54. Vermeulen J, Gosselink M, Hop W, Lange J, Coene P, Van der Harst E, et al. Hospital mortality after emergency surgery for perforated diverticulitis. Ned Tijdschr Geneesk 2009;153:1209-14.

55. Mäkelä J, Kiviniemi H, Laitinen S. Prevalence of Perforated Sigmoid Diverticulitis Is Increasing. Dis Colon Rectum 2002;45:955-61.

56. Frileux P, Dubrez J, Burdy G, Roullet-Audy J, Dalban-
Sillas B, Bonnaventure F, et al. Sigmoid diverticulitis. Longitudinal analysis of 222 patients with a minimal follow up of 5 years. Colorectal Dis. 2010;12:674-80.

57. Landen S, Nafteaux P. Primary Anastomosis and Diverting Colostomy in Diffuse Diverticular Peritonitis. Acta Chir Belg. 2002;102:24-9.

58. Holmer C, Lehmann K, Gröne J, Buhr H, Ritz J. Perforationsrisiko und Patietenalter. Eine Risikoanalyse bei der akuten Sigmadivertikulitis. Chirurg. 2011;82:35966.

59. Hequera J, Avella G, Obregón G. Peritonitis diverticular. Rev Mex Coloproctol. 2005;11:23-32.

60. López DE, Brown BR. Diverticulitis: The most common colon emergency for the acute care surgeon. Scand J Surg. 2010;99:86-9.

61. Vermeulen J, Gosselink M, Busschbach J, Lange J. Avoiding or Reversing Hartmann's Procedure Provides Improved Quality of Life After Perforated Diverticulitis. J Gastrointest Surg. 2010;14:651-7.

62. López F, Norero E, Paulos A, Anuch C, Márquez S, Molina ME, y cols. Operación de Hartmann y su posterior reconstitución realizadas por un mismo equipo quirúrgico. Rev Chil Cir. 2003;55;591-7.

63. Bannura G, Contreras J, Melo C, Barrera A, Soto D, Mansilla J. Indicaciones y resultados alejados del tratamiento quirúrgico electivo de la enfermedad diverticular de colon sigmoides. Rev Med Chile 2005;133:1037-42. 